\title{
ON SPECTRAL RADIUS ALGEBRAS
}

\author{
Animikh Biswas, Alan Lambert, SRdjan Petrovic \\ AND BARNET WEINSTOCK
}

\begin{abstract}
We show how one can associate a Hermitian operator $P$ to every operator $A$, and we prove that the invertibility properties of $P$ imply the non-transitivity and density of the spectral radius algebra associated to $A$. In the finite dimensional case we give a complete characterization of these algebras in terms of $P$. In addition, we show that in the finite dimensional case, the spectral radius algebra always properly contains the commutant of $A$.
\end{abstract}

Mathematics subject classification (2000): 47A15, 47A65, 47B15.

Key words and phrases: Spectral radius algebras, invariant subspaces, operators on finite dimensional spaces.

\section{REFERENCES}

[1] A. BISWAS, A. LAMBERT, AND S. PETROVIC, On spectral radius algebras and normal operators, Indiana University Mathematics Journal 56 (2007), no. 4, 1661-1674.

[2] A. LAMBERT, S. PETROVIC, Beyond hyperinvariance for compact operators, J. Funct. Anal. 219 (2005), no. $1,93-108$.

[3] A. SHIELDS, Weighted shift operators and analytic function theory, in "Topics in Operator Theory", pp. 49-128, Mathematical Surveys No. 13, Amer. Math. Soc., Providence, R.I., 1974. 